Research Article

\title{
SPSS Analysis of Pain Factors in Rotator Cuff Repair
}

\author{
Yi Zhou, ${ }^{1}$ Huali Chen $\mathbb{D}^{2}{ }^{2}$ Jing Wang, ${ }^{1}$ Hui Wu, ${ }^{1}$ Yuanjie Zeng, ${ }^{1}$ Xiaohui Yi, \\ and Yan Zhang $\mathbb{D}^{3,4}$ \\ ${ }^{1}$ Department of Orthopedics (Department of Osteoarticular and Sports Medicine), \\ Hunan Provincial People's Hospital (The First-Affiliated Hospital of Hunan Normal University), Changsha 410005, China \\ ${ }^{2}$ Quality Control Office of Nursing Department, \\ Hunan Provincial People's Hospital (The First-Affiliated Hospital of Hunan Normal University), Changsha 410005, China \\ ${ }^{3}$ Department of Cardiovascular Internal Medicine, \\ Hunan Provincial People's Hospital (The First-Affiliated Hospital of Hunan Normal University), Changsha 410005, China \\ ${ }^{4}$ Hunan Provincial Heart Failure Clinical Medical Research Center, \\ Hunan Provincial People's Hospital (The First-Affiliated Hospital of Hunan Normal University), Changsha 410005, China
}

Correspondence should be addressed to Yan Zhang; zhangyan.01@outlook.com

Received 4 September 2021; Accepted 15 September 2021; Published 29 September 2021

Academic Editor: Weiguo Li

Copyright ( 2021 Yi Zhou et al. This is an open access article distributed under the Creative Commons Attribution License, which permits unrestricted use, distribution, and reproduction in any medium, provided the original work is properly cited.

In this study, the matched case-control study was used. Nineteen cases of severe pain in the early postoperative period among 55 patients were set as the observation group, and 57 cases of simultaneous rotator cuff repair without severe pain were matched in a $1: 3$ ratio as the control group. Patients' general information, disease characteristics, anesthesia and analgesia scheme, and operation information were collected. Frequency statistics, Wilcoxon signed rank sum test, regulatory effect analysis, and Poisson regression analysis were performed on these data. Some findings are included in the analysis. (1) There was a markable difference of 0.01 between the preoperative $48 \mathrm{~h}$ maximum pain value and the postoperative $48 \mathrm{~h}$ maximum pain value in the observation group. (2) There was a markable difference of 0.01 between the size and shape of the wound tear and the maximum pain value at $48 \mathrm{~h}$ after operation in the observation group. (3) When the number of opioid use affected the maximum pain value at $48 \mathrm{~h}$ after operation, the regulatory variables (type, quantity, and number of days of postoperative analgesics) were at different levels, and the impact amplitude had markable differences. (4) Age has a markable negative impact on the postoperative hospital stay. (5) The operation duration has a markable positive relationship with the postoperative hospital stay. (6) The analgesic pump had a markable positive impact on the postoperative hospital stay. (7) The location of injury did not affect the postoperative hospital stay.

\section{Introduction}

Rotator cuff suture reduces the postoperative adhesion of the shoulder joint in wound treatment and can restore the shoulder joint function of patients to the maximum degree $[1,2]$. Compared with the previous incision treatment, the rotator cuff suture has the advantages of less trauma [3], smaller incision, and more beautiful appearance after operation $[4,5]$.

SPSS is very useful in data analysis [6]. The pain problem before and after surgery has been widely discussed by scholars, and mathematical analysis is gradually used more frequently [7-9]. Many studies have discussed rotator cuff sutures with the method of mathematical analysis [10-12]. There are many other studies that have been conducted prospectively in patients with rotator cuff sutures $[13,14]$. Derwin and Sahoo [15] studied factors such as the number of suture anchors used in rotator cuff repair and pain. Some researchers have described the magnitude of pain after rotator cuff suture [16]. Pain-related studies in rotator cuff sutures also include predictions of surgical outcomes and discussion of the size and shape of a patient's wound $[17,18]$.

The above observations indicate that the pain factors in patients undergoing rotator cuff repair deserve further 
analysis. In this work, we analyzed preoperative and postoperative pain in 55 patients to observe disease characteristics, anesthesia and analgesia regimens, operative information, and so on. SPSS software was used for frequency statistics, Wilcoxon signed rank sum test, adjustment effect analysis, and Poisson regression analysis. This work can further discuss the situation of pain factors in rotator cuff sutures.

\section{Objective and Methods}

The statistic analysis of the frequency for 55 patients is given in Table 1.

Table 1 provides the results of the frequency analysis for 55 patients. The original information is shown in Supplementary Materials. SPSS was used to analyze the results. As can be seen from the above table, on the distribution of the affected side in the observation group, most of the samples were "right shoulder," and the proportion was $76.92 \%$. The proportion of "women" in the observation group was $53.85 \%$ and that of the male sample was $46.15 \%$. The proportion of "right shoulder" in the control group was $64.29 \%$. There was also left shoulder in $35.71 \%$ of samples. The highest proportion of females in the control group was 69.05\%. 30.95\% of the samples were male.

\section{Results and Discussion}

The paired sample Wilcoxon analysis results are given in Table 2

It can be seen from Table 2 that the Wilcoxon signed rank-sum test is used to study the differences of experimental data. Among the two paired datasets, one actually presented a difference $(p<0.05)$. The specific analysis shows that there is a markable difference of 0.01 level between the presurgery and postsurgery $48 \mathrm{~h}$ maximum pain values in the observation group $(p<0.01)$, and the specific comparison difference shows that the presurgery median value (3.000) in the observation group is markablely lower than the median value of postsurgery $48 \mathrm{~h}$ maximum pain value (8.000). Therefore, the observation group data were true and valid. Next, the observation group data were analyzed.

It can be seen from Table 3 that the Wilcoxon signed rank-sum test is used to study the differences of experimental data. The paired data of the two groups showed differences $(p<0.05)$. The specific analysis showed that there was a markable difference of 0.01 between the wound tear size and the maximum pain $48 \mathrm{~h}$ after surgery in the observation group $(p=0.003<0.01)$. By specific comparison, the median tear size (1.500) was markablely lower than the median maximum pain $48 \mathrm{~h}$ after surgery (8.000).

There was a markable difference at the 0.01 level between wound tear shape and wound retraction $(p=0.009<0.01)$, and the median tear shape (2.000) was markablely higher than the median retraction.

A total of 2 sets of paired data will all present differences.

As given in Table 4, some information of patients is missing the variables to be investigated in this analysis, so $n=13$. The regulatory effects were divided into three models, with the independent variable (opioid use times) included in model 1. Model 2 included regulatory variables (type, quantity, and number of days of postoperative analgesics) on the basis of model 1, and model 3 included interactive terms (the product term of independent variables and regulatory variables) on the basis of model 2 .

Model 1 was used for studying the effect of independent variable (opioid use times) on dependent variable (maximum pain $48 \mathrm{~h}$ after surgery) without considering the interference of regulatory variables (type, quantity, and number of days of postoperative analgesic). As given in the above table, the independent variable (number of opioid use) showed markable $(t=0.696, p=0.501>0.05)$. This means that, regardless of the effects of regulatory variables (type, quantity, and number of days of postoperative analgesics), the number of opioid use did not have a markable impact on the maximum pain value at $48 \mathrm{~h}$ after surgery, which should still be investigated for further regulatory effects.

The adjustment effect can be viewed in two ways. The first way is to view the significance of $F$ value change from model 2 to model 3 . The second way is to check the significance of the interaction items in model 3. The regulation effect is analyzed in the second way this time.

As given in the above table, the interaction between the number of opioid use and the type, quantity, and number of days of postoperative analgesics was statistically markable $(t=-2.874, p=0.018<0.05)$. It meant that when the number of opioid use affected the maximum pain value at $48 \mathrm{~h}$ after operation, the magnitude of impact was markablely different when the regulatory variables (type, quantity, and number of days of postoperative analgesic drugs) were at different levels.

As given in Table 5, age, operation duration, analgesic pump, and injury site in the observation group were regarded as independent variables, while the postoperative hospital stay was regarded as dependent variable for Poisson regression analysis. Only 10 cases met the requirements when the data of age, operation time, analgesic pump, and injury site of the observation group were available. Therefore, only these ten cases were analyzed. The model pseudo$R$ formula (McFadden $R$ formula) was 0.487 , which meant that age, operation duration, analgesic pump, and injury site could explain $48.7 \%$ of the change in the postoperative hospital stay. It can be seen from the above table that the model formula was $\log (u)=5.979-0.122^{*}$ age $+0.015^{*}$ operation duration $+1.513^{*}$ analgesic pump $-0.045^{*}$ injury site (where $U$ represents the expected mean).

The regression coefficient value of age was -0.122 and showed a markable value at 0.01 level $(Z=-5.394, p<0.01)$, which meant that age had a markable negative impact on the postoperative hospital stay. An OR of 0.885 meant a 0.885 fold change in postoperative hospital stay as age increased by one unit.

The regression coefficient value of the operation duration was 0.015 , and the significance was shown at 0.05 level $(z=2.260, p=0.024<0.05)$, indicating that the operation duration had a markable positive impact on the postoperative hospital stay. An OR of 1.015 indicated a 1.015-fold increase in postoperative hospital stay with a unit increase in procedure duration. 
TABle 1: Statistic analysis of the frequency for 55 patients.

\begin{tabular}{|c|c|c|c|}
\hline Name & Option & Frequency & Percentage $(\%)$ \\
\hline \multirow{2}{*}{ Affected side of the observation group $(n=13)$} & Right shoulder & 10 & 76.92 \\
\hline & Left shoulder & 3 & 23.08 \\
\hline \multirow{2}{*}{ Gender $(n=13)$} & Woman & 7 & 53.85 \\
\hline & Man & 6 & 46.15 \\
\hline \multirow{2}{*}{ Affected side of the control group $(n=42)$} & Right shoulder & 27 & 64.29 \\
\hline & Left shoulder & 15 & 35.71 \\
\hline \multirow{2}{*}{ Gender $(n=42)$} & Woman & 29 & 69.05 \\
\hline & Man & 13 & 30.95 \\
\hline \multicolumn{3}{|c|}{ Add up to } & 100.0 \\
\hline
\end{tabular}

TABLE 2: Paired sample Wilcoxon analysis results.

\begin{tabular}{|c|c|c|c|c|c|}
\hline \multirow[t]{2}{*}{ Name } & \multicolumn{2}{|c|}{$\begin{array}{c}\text { Paired } \\
\text { (median) }\end{array}$} & \multirow{2}{*}{$\begin{array}{c}\text { Difference } \\
\text { (pair 1-pair 2) }\end{array}$} & \multirow{2}{*}{$\begin{array}{l}\text { Statistical } \\
\text { value, } z\end{array}$} & \multirow[t]{2}{*}{$P$} \\
\hline & Pair 1 & Pair 2 & & & \\
\hline Maximum pain $48 \mathrm{~h}$ after preoperative pairing in the observation group & 3.000 & 8.000 & -5.000 & 3.208 & $\leq 0.001^{* *}$ \\
\hline $\begin{array}{l}\text { Patients in the control group were paired with the maximum pain value at } 48 \mathrm{~h} \text { after } \\
\text { operation }\end{array}$ & 3.000 & 3.000 & 0.000 & 0.493 & 0.622 \\
\hline
\end{tabular}

${ }^{* *} P<0.01$.

TABle 3: Paired sample Wilcoxon analysis results.

\begin{tabular}{lcccc}
\hline Name & Paired (median) & \multicolumn{2}{c}{$\begin{array}{c}\text { Difference } \\
\text { Statistical } \\
\text { value, z }\end{array}$} & P \\
\hline $\begin{array}{l}\text { The size of wound tear in the observation group was paired with the } \\
\text { maximum pain value at 48 h after operation }\end{array}$ & 1.500 & 8.000 & -6.500 & 2.944 \\
Does the tear shape pair retract & 2.000 & 0.000 & 2.000 & $2.003^{* *}$ \\
\hline
\end{tabular}

${ }^{* *} P<0.01$.

TABLE 4: Adjustment effect analysis results $(n=13)$.

\begin{tabular}{|c|c|c|c|c|c|c|c|c|c|}
\hline & \multicolumn{3}{|c|}{ Model 1} & \multicolumn{3}{|c|}{ Model 2} & \multicolumn{3}{|c|}{ Model 3} \\
\hline & $\begin{array}{c}\text { Standard } \\
\text { error }\end{array}$ & $t$ & $P$ & $\begin{array}{c}\text { Standard } \\
\text { error }\end{array}$ & $t$ & $P$ & $\begin{array}{l}\text { Standard } \\
\text { error }\end{array}$ & $t$ & $P$ \\
\hline Constant & 0.184 & 41.298 & $\leq 0.001^{* *}$ & 0.191 & 39.78 & $\leq 0.001^{* *}$ & 0.147 & 51.686 & $\leq 0.001^{* *}$ \\
\hline Number of opioid uses & 0.103 & 0.696 & 0.501 & 0.107 & 0.714 & 0.492 & 0.092 & -0.488 & 0.637 \\
\hline $\begin{array}{l}\text { Type, quantity, and number of days of } \\
\text { postoperative analgesic }\end{array}$ & & & & 0.066 & 0.455 & 0.659 & 0.056 & -0.665 & 0.523 \\
\hline $\begin{array}{l}\text { Number of opioid use*, type, quantity, } \\
\text { and number of days of postoperative } \\
\text { analgesics }\end{array}$ & & & & & & & 0.028 & -2.874 & $0.018^{*}$ \\
\hline$R^{2}$ & \multicolumn{3}{|c|}{0.042} & \multicolumn{3}{|c|}{0.062} & \multicolumn{3}{|c|}{0.511} \\
\hline Adjust $r$ & \multicolumn{3}{|c|}{-0.045} & \multicolumn{3}{|c|}{-0.126} & \multicolumn{3}{|c|}{0.347} \\
\hline Variance ratio & \multirow{2}{*}{\multicolumn{3}{|c|}{$F(1,11)=\underset{0.485, p=0.501}{0.042}$}} & \multicolumn{3}{|c|}{$F(2,10)=0.328, p=0.728$} & \multicolumn{3}{|c|}{$F(3,9)=3.130, p=0.080$} \\
\hline$\triangle R^{2}$ & & & & \multirow{2}{*}{\multicolumn{3}{|c|}{$F(1,10)=0.207, p=0.659$}} & \multirow{2}{*}{\multicolumn{3}{|c|}{$\begin{array}{c}0.449 \\
F(1-9)-8258\end{array}$}} \\
\hline$\triangle F$ value & \multicolumn{3}{|c|}{$F(1,11)=0.485, p=0.501$} & & & & & & $=0.018$ \\
\hline
\end{tabular}

Dependent variable: maximum pain $48 \mathrm{~h}$ after surgery in the observation group. ${ }^{*} P<0.05 .{ }^{* *} P<0.01$.

The regression coefficient value of the analgesic pump was 1.513, and the significance was shown at 0.01 level $(z=3.212, p<0.01)$, which meant that the analgesic pump had a markable positive impact on the postoperative hospital stay. An odds ratio (OR value) of 4.540, which means a 4.540-fold change in postoperative hospital days, occurred when the analgesic pump was increased by one unit.
The regression coefficient value for the injury site was -0.045 , but it was not markable $(z=-0.195$, $p=0.845>0.05)$, suggesting that the injury site did not affect the postoperative hospital stay.

According to the summary and analysis, it can be seen that the operation duration and analgesic pump have a markable positive impact on the postoperative hospital 
TABle 5: Summary of Poisson regression analysis results $(n=10)$.

\begin{tabular}{|c|c|c|c|c|c|c|}
\hline Item & Coefficient of regression & Standard error & $Z$ value & $P$ value & OR value & OR value, $95 \% \mathrm{CI}$ \\
\hline Observation group age & -0.122 & 0.023 & -5.394 & $\leq 0.001^{* *}$ & 0.885 & $0.846-0.925$ \\
\hline Duration of surgery & 0.015 & 0.006 & 2.260 & 0.024 & 1.015 & $1.002-1.028$ \\
\hline Analgesic pump & 1.513 & 0.471 & 3.212 & $\leq 0.001^{* *}$ & 4.540 & $1.804-11.428$ \\
\hline Injury site & -0.045 & 0.232 & -0.195 & 0.845 & 0.956 & $0.606-1.507$ \\
\hline Intercept & 5.979 & 1.668 & 3.584 & $\leq 0.001^{* *}$ & 395.129 & $15.024-10391.750$ \\
\hline
\end{tabular}

Dependent variable: postoperative hospital stay in the observation group. McFadden $R$ formula, 0.487 .

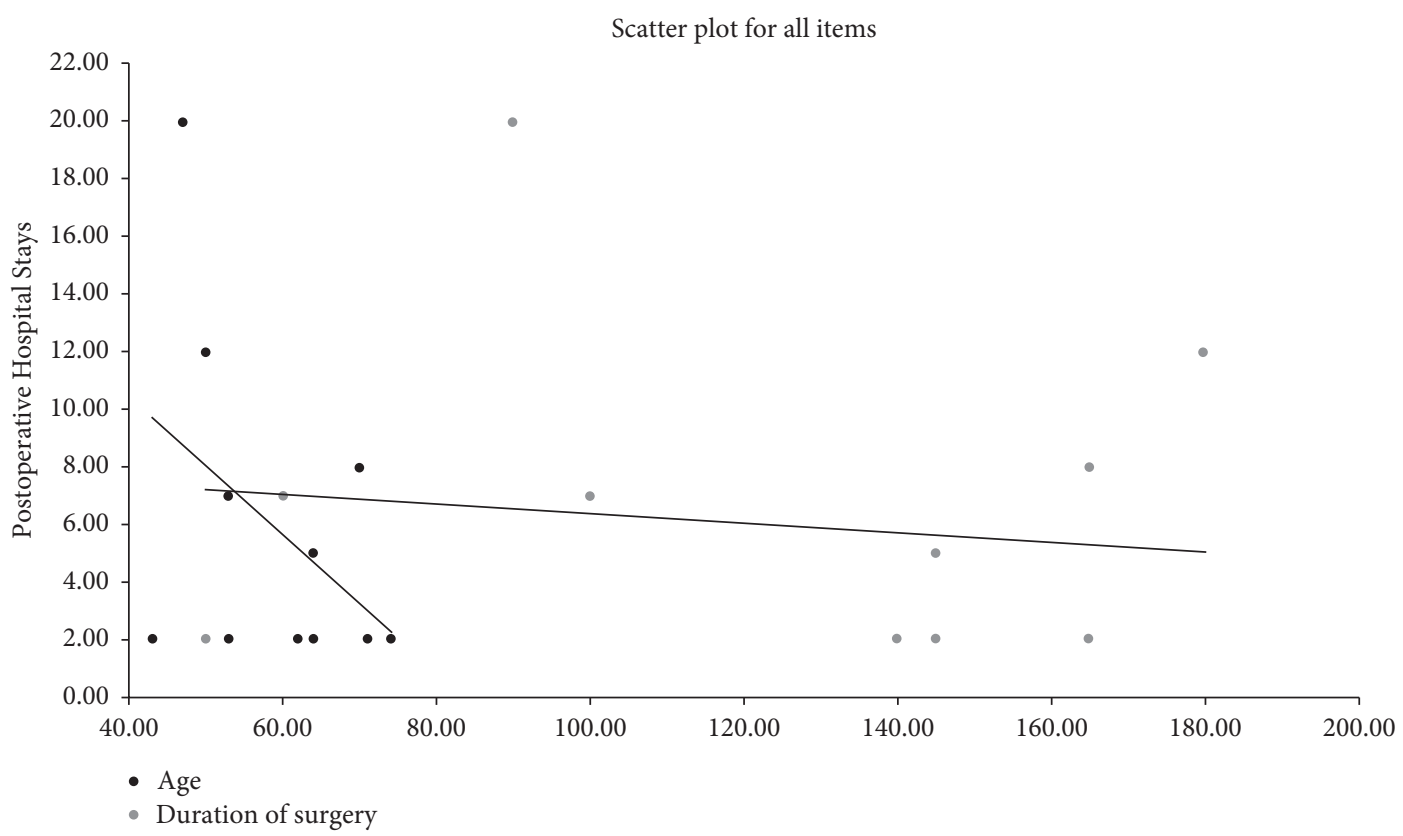

FIGURE 1: Scatter plot for 3 items.

stay, and age has a markable negative impact on the postoperative hospital stay. However, the injury site did not affect the postoperative hospital stay. The scatter plot of age, operation duration (analgesia pump was not good for making trend chart, so it was excluded), and postoperative hospital days in the observation group is shown in Figure 1.

\section{Conclusions}

Data analysis was performed on 55 patients. In the matched case-control study, 19 cases with severe pain in the early postoperative period were set as the observation group, and 57 cases without severe pain who underwent rotator cuff repair at the same time were matched according to the ratio of $1: 3$ as the control group. The general data, disease characteristics, anesthesia and analgesia scheme, and operation information of the patients were collected. The data were subjected to frequency statistics, Wilcoxon signed rank-sum test, adjustment effect analysis, and Poisson regression analysis. Some findings were included in the analysis. (1) There was a markable difference of 0.01 between the preoperative $48 \mathrm{~h}$ maximum pain value and the postoperative $48 \mathrm{~h}$ maximum pain value in the observation group. (2) There was a markable difference of 0.01 between the size and shape of the wound tear and the maximum pain value at $48 \mathrm{~h}$ after operation in the observation group. (3) When the number of opioid use affected the maximum pain value at $48 \mathrm{~h}$ after operation, the regulatory variables (type, quantity, and number of days of postoperative analgesics) were at different levels, and the impact amplitude had markable differences. (4) Age has a markable negative impact on the postoperative hospital stay. (5) The operation duration has a markable positive relationship with the postoperative hospital stay. (6) The analgesic pump had a markable positive impact on the postoperative hospital stay. (7) The location of injury did not affect the postoperative hospital stay.

\section{Data Availability}

The data used to support the findings of this study are included within the article and the Supplementary Materials.

\section{Ethical Approval}

Ethical approval for this work was obtained from the Ethical Review Committee of Hunan Provincial People's Hospital (the First Affiliated Hospital of Hunan Normal University). 


\section{Conflicts of Interest}

The authors declare that they have no conflicts of interest.

\section{Acknowledgments}

This work was supported by Health Commission of Hunan Province, China (Project No. 202104072141).

\section{Supplementary Materials}

Raw data for the analysis. (Supplementary Materials)

\section{References}

[1] R. Prasetia, B. Sukhapradit, and B. Chernchujit, "Clinical features and repair integrity after knotless-in situ suture bridge technique in high-grade bursal side rotator cuff tears," Journal of Orthopaedics, vol. 20, pp. 352-358, 2020.

[2] N. A. Zurita Uroz, F. Abat, and A. Calvo Diaz, "All-suture repair for compressive rotator cuff tears: reducing the traction of the tissue," Arthroscopy Techniques, vol. 6, no. 2, pp. e499-e503, 2017.

[3] H. Van der Bracht, T. Van den Langenbergh, M. Pouillon, S. Verhasselt, P. Verniers, and D. Stoffelen, "Rotator cuff repair with all-suture anchors: a midterm magnetic resonance imaging evaluation of repair integrity and cyst formation," Journal of Shoulder and Elbow Surgery, vol. 27, no. 11, pp. 2006-2012, 2018.

[4] M. P. McCabe, "Editorial commentary: choose wisely: rotator cuff all-suture anchors," Arthroscopy: The Journal of Arthroscopic \& Related Surgery, vol. 36, no. 11, pp. 2812-2813, 2020.

[5] J. Buckup, D. Smolen, F. Hess, C. Sternberg, and J. Leuzinger, "The arthroscopic triple-row modified suture bridge technique for rotator cuff repair: functional outcome and repair integrity," Journal of Shoulder and Elbow Surgery, vol. 29, no. 2, pp. 308-315, 2020.

[6] Q. Tan and W. Shao, "Investigation on Health promotion by the typical sports for teenagers with self-efficacy and sports commitment questionnaires," Evidence-Based Complementary and Alternative Medicine: eCAM, vol. 2021, Article ID 8677182, 7 pages, 2021.

[7] M. A. Röling, B. Hesseling, N. M. C. Mathijssen, and R. M. Bloem, "Hip arthroscopy for femoroacetabular impingement syndrome results in 2 recovery patterns based on preoperative pain and on arthritis: improvers and non-improvers," Arthroscopy, Sports Medicine, and Rehabilitation, 2021.

[8] K. Powezka, T. Adjei, W. von Rosenberg et al., "A pilot study of preoperative heart rate variability predicting pain during local anesthetic varicose vein surgery," Journal of Vascular Surgery: Venous and Lymphatic Disorders, vol. 7, no. 3, pp. 382-386, 2019.

[9] X. He, L. Chen, H. Chen, Y. Feng, B. Zhu, and C. Yang, "Diagnostic accuracy of procalcitonin for bacterial infection in liver failure: a meta-analysis," Bioinorganic Chemistry and Applications, vol. 2021, Article ID 5801139, 8 pages, 2021.

[10] G. Villatte, R. Erivan, J. Barth, N. Bonnevialle, S. Descamps, and S. Boisgard, "Progression and projection for shoulder surgery in France, 2012-2070: epidemiologic study with trend and projection analysis," Orthopaedics \& Traumatology: Surgery \& Research, vol. 106, no. 6, pp. 1067-1077, 2020.

[11] M. Liu, X. Lin, Q. Tan, and X. Han, "Evidence-based analysis of the emergency temporary cardiac pacing (electrical stimulation from metal wire electrode)," Bioinorganic Chemistry and Applications, vol. 2021, Article ID 5677598, 9 pages, 2021.

[12] J. Johnson, M. Pinto, E. Brabston et al., "Attitudes and awareness of suture anchor cost: a survey of shoulder surgeons performing rotator cuff repairs," Journal of Shoulder and Elbow Surgery, vol. 29, no. 3, pp. 643-653, 2020.

[13] R. Dukan, P. Ledinot, J. Donadio, and P. Boyer, "Arthroscopic rotator cuff repair with a knotless suture bridge technique: functional and radiological outcomes after a minimum follow-up of 5 years," Arthroscopy: The Journal of Arthroscopic \& Related Surgery, vol. 35, no. 7, pp. 2003-2011, 2019.

[14] P. Belón-Perez and A. I. Cuesta-Vargas, "Immediate effects of thoracic spine manipulation upon shoulder functionality in patients with sutured rotator cuff repair: a prospective study," Journal of Manipulative and Physiological Therapeutics, vol. 41, no. 7, pp. 589-595, 2018.

[15] K. A. Derwin, S. Sahoo, A. Zajichek et al., "Tear characteristics and surgeon influence repair technique and suture anchor use in repair of superior-posterior rotator cuff tendon tears," Journal of Shoulder and Elbow Surgery, vol. 28, no. 2, pp. 227-236, 2019.

[16] K. Yamakado, “A prospective randomized trial comparing suture bridge and medially based single-row rotator cuff repair in medium-sized supraspinatus tears," Arthroscopy: The Journal of Arthroscopic \& Related Surgery, vol. 35, no. 10, pp. 2803-2813, 2019.

[17] D. Goutallier, J.-M. Postel, X. Chevalier, J. Beaudreuil, and S. Zilber, "Intermediate term functional outcome prediction following full thickness rotator cuff tear reparative or not reparative surgery," Orthopaedics \& Traumatology: Surgery \& Research, vol. 96, no. 7, pp. 727-733, 2010.

[18] E. Buess, K.-U. Steuber, and B. Waibl, "Open versus arthroscopic rotator cuff repair: a comparative view of 96 cases," Arthroscopy: The Journal of Arthroscopic \& Related Surgery, vol. 21, no. 5, pp. 597-604, 2005. 